\title{
The Swiss Society of Experimental Pharmacology in Times of Change
}

\author{
Gabriele Weitz-Schmidt*a and Urs Rüegg ${ }^{\mathrm{b}}$
}

\begin{abstract}
Experimental pharmacology is undergoing fundamental changes. This article describes the challenges and opportunities associated with these changes from the perspective of the Swiss Society of Pharmacology (SSEP), the society which aims to advance experimental pharmacology in Switzerland and abroad.
\end{abstract}

Keywords: Experimental pharmacology · Swiss Society of Experimental Pharmacology (SSEP)

\section{SSEP}

\section{What is Experimental Pharmacology and What Is it Good for?}

Etymologically, pharmacology is the science of drugs (Greek: pharmakon, medicine or drug; and logos, study or reason). Within pharmacology, the field of experimental pharmacology investigates the mechanisms of action and effects of chemically or biologically produced compounds and other therapeutically active modalities, such as RNA-based derivatives, as well as cell and gene therapies, on biological systems, both qualitatively and quantitatively. Experimental pharmacology is at the core of the drug discovery process. The concepts of 'understanding disease at the molecular level', 'validating therapeutic targets', 'establishing structure-activity relationships' and 'assessing concentration-response relationships' for innovative drugs are fundamental to this discipline. Throughout the drug discovery process, experimental pharmacologists work in close partnership with chemists, biologists, physicians and regulatory experts, among experts of other disciplines involved in the development of pioneering therapies.

Historically, the task of experimental pharmacologists was to explore and document effects of herbal and animal extracts and minerals on living organisms (Fig. 1). These explorations tended to be neither systematic nor were they necessarily based on scientific principles. In the mid-19 ${ }^{\text {th }}$ century, the adoption of scientific methods, i.e. the learning from evidence generated by systematic, hypothesis-driven trial and error, constituted a historical turning point in the discovery and development of novel medicines, laying the foundation of experimental pharmacology as we understand it today. For many decades, experimental pharmacology largely focussed on the study of substance-drug receptor interactions. The deliverable of this pharmacological research is a huge armamentarium of well-characterized drugs and medications, which revolutionized medicine in the last century and resulted in historically unprecedented therapeutic success across medical disciplines.
By the beginning of the $21^{\text {st }}$ century, it is becoming evident that the above-mentioned paradigm of experimental pharmacology, as successful as it has been in the past, is being transformed fundamentally once more: Today's experimental pharmacologist must not only have an excellent foundation in the quantitative aspects of the field, yet also the ability to apply a holistic approach to the drug discovery and drug development process. This 'holism' has several dimensions: Firstly, experimental pharmacology must no longer restrict itself to the development of 'drugs' in the historical sense. Modern therapeutic interventions encompass ever wider scopes of modalities outside historical boundaries, be it RNA-based, cell or gene therapies, to just name a few examples. Typically, these interventions are associated with historically unprecedented levels of complexity to be dealt with by experimental pharmacologists. For example, starting materials of live cell products are highly variable, difficult to characterize and their mechanisms of action are understood only partially. Secondly, nowadays' experimental pharmacologists need to master the challenge of bridging their detailed knowledge

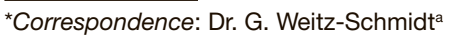
E-mail: gabriele.weitz@allocyte-pharma.com aAlloCyte Pharmaceuticals Basel

bProf. em., Universities Geneva and Lausanne

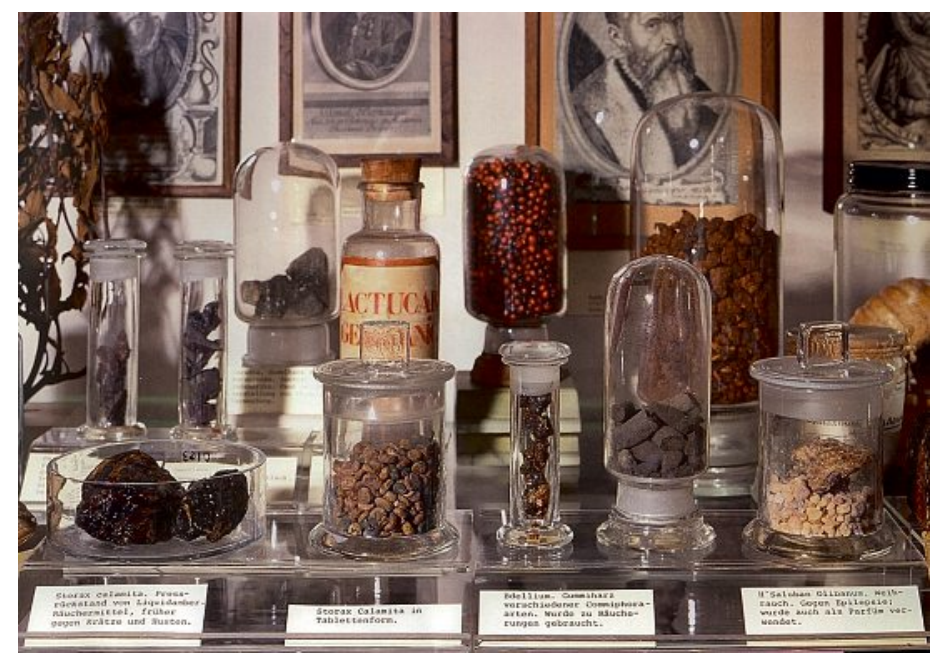

Fig. 1. Materia medica: The term 'Materia medica' derives from the title of a work by the Greek physician Pedanius Dioscorides in the $1^{\text {st }}$ century $A D$, 'De Materia Medica'. De Materia Medica describes many drugs derived from herbs, animals and mineral substances. Picture courtesy of Dr. Michael Kessler, Head of the Pharmacy Museum of the University Basel. 
of pharmacological mechanisms at molecular and cellular levels with a perspective on the organism as a whole. The unprecedented challenge comes with unprecedented therapeutic promise: Supported by computerized technologies, a 'holistic' experimental pharmacology holds the promise of translating molecular knowledge into innovatively integrated therapeutic concepts, maximizing individual patient's therapeutic benefit and mitigating associated risk to a minimum. In the light of these challenges and opportunities as well as in the light of the rising number of medications (e.g. the total number of medical prescriptions dispensed in the United States of America from 2009 to 2015 increased from 3.95 to 4.37 billion), ${ }^{[1]}$ the need for well-trained experimental pharmacologists with a broad and open-minded perception of their profession, embracing innovation in times of radical change, has never been greater.

Switzerland is one of the world's leading countries in the discovery, development and production of ground-breaking pharmaceuticals, reflected by the presence of established pharmaceutical companies as well as innovation-driven biotech startup companies. These unique settings and the breadth of expertise in Switzerland, supported by excellent educational programs of pharmacological research, pharmaceutical development and safety evaluation provides a sound basis for continued international competitiveness and economic success. It is probably fair to state that Switzerland has excellent chances to emerge as 'the' place to advance experimental pharmacology to its next level.

\section{The SSEP in Brief}

The Swiss Society of Experimental Pharmacology is an organisation that represents professionals working across all fields of experimental pharmacology. Its objective is to defend and promote the interests of experimental pharmacologists in Switzerland and to advance scientific and translational research in the field. The SSEP is a daughter society of the Swiss Society of Pharmacology and Toxicology (SSPT) and affiliated with the Swiss Academy of Natural Sciences (SCNAT), the International Union of Basic and Clinical Pharmacology (IUPHAR) and the Federation of European Pharmacological Societies (EPHAR). Furthermore, the SSEP connects to the field of Life Sciences via a partnership with Life Sciences Switzerland (LS2) (Fig. 2). Traditionally, the SSEP participates in the organisation of the Annual Spring Meeting of the SSPT at which the Novartis Prize for the best talk and best poster by young investigators is awarded and every second year the 'Bürgi Prize' winner is honoured. This prize is named after Emil Bürgi who was director of the Pharmacological Institute of the University Bern from 1906-1942 and is awarded to young scientists for excellent $\mathrm{PhD}$ theses with a pharmacological content.[2] Furthermore, the SSEP regularly organizes a scientific symposium covering

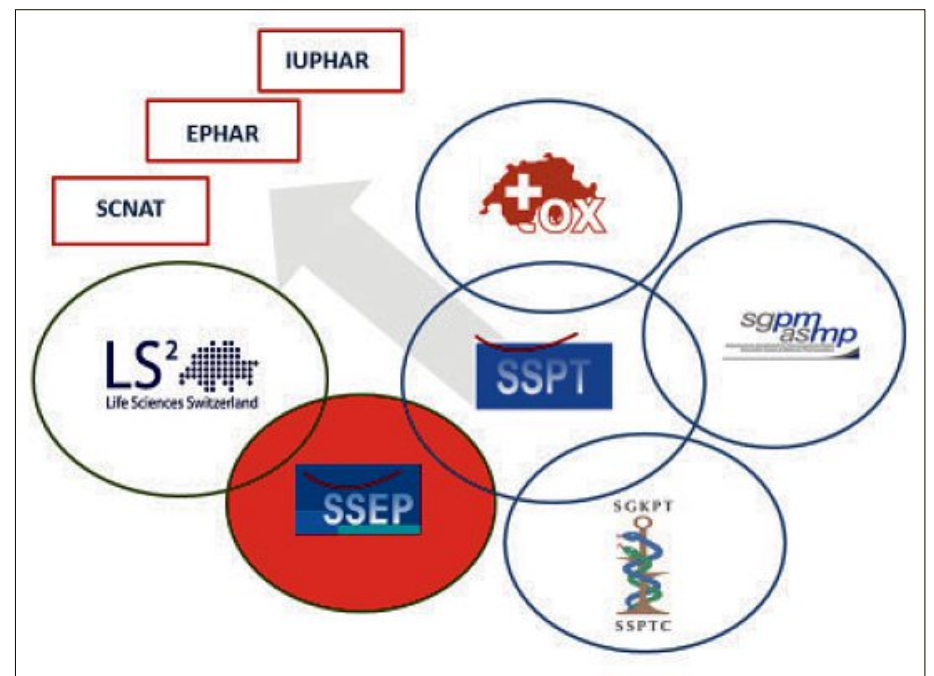

Fig. 2. SSEP Network. The SSEP is a 'daughter' society of the SSPT. Further, the SSEP connects to the field of life sciences via a partnership with LS2. The SSEP is affiliated via SSPT with EPHAR, SCNAT, and IUPHAR. SSEP: Swiss Society of Experimental Pharmacology; SSPT: Swiss Society of Pharmacology and Toxicology; Tox: Swiss Society of Toxicology, SGKPT/SSPTC: Swiss Society of Clinical Pharmacology and Toxicology; LS2: Life Sciences Switzerland; sgpm/asmp: Swiss Society of Pharmaceutical Medicine; SCNAT: Swiss Academy of Natural Sciences; EPHAR: Federation of European Pharmacological Societies; IUPHAR: International Union of Basic and Clinical Pharmacology.

key developments in the field at the annual conferences organized by the LS2.

Membership in the SSEP provides a number of important benefits to professionals actively involved or interested in experimental pharmacology:

- Networking opportunities with experimental pharmacologists in Switzerland and around the world.

- Participation in regular scientific meetings organised by the SSPT and LS2 at reduced fees.

- Travel grants awards to young investigators (PhD students and, more recently, post-docs).

The society is proud to represent more than 100 pharmacologists from academia and industry. The SSEP headquarters are located in Bern at the Institute of Pharmacology of the University Bern.

\section{History of the SSEP}

The history of the SSEP is closely related to the history of the SSPT, because the SSEP emerged from the SSPT in 2005. Briefly, the SSPT was founded in 1965. Peter Waser, director of the Institute of Pharmacology of the University Zurich from 1963-1988, formed a preliminary council of the SSPT at an assembly in November 1964 (Fig. 3). The first society meeting was held in Basel in May 1965 with about 30 members and, at that point, the society took off. [3] The founding date of 1965 positions the SSPT among the younger pharmacological societies worldwide; in comparison, the American Society of Pharmacology and Experimental Therapeutics was founded 1908 and the British Pharmacological Society in 1931. In laying down the by-laws of the SSPT,

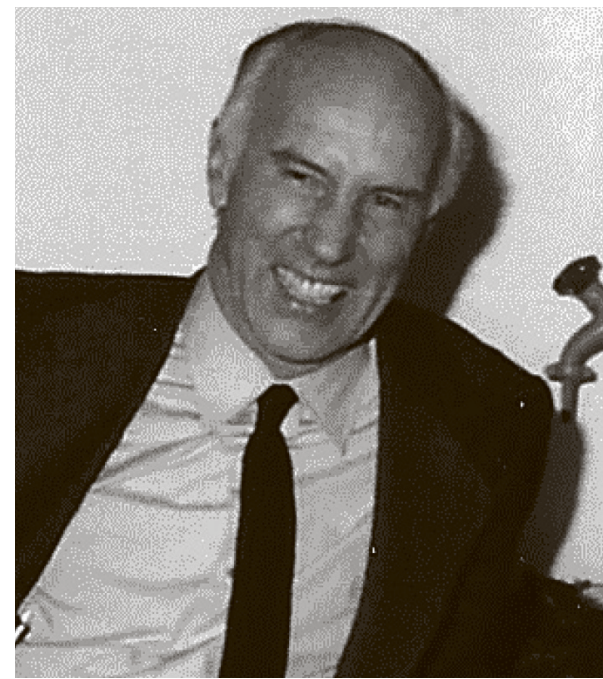

Fig. 3. SSPT founder professor Peter G. Waser, Director of the Pharmacological Institute of the University of Zurich from 1963-1988, in the 1970s. 
the founder agreed that: "The aim of the SSPT shall be to defend and integrate the interests of pharmacology and toxicology in education and research in Switzerland, and to connect with other societies abroad having similar interests."

The annual meetings were held in turn in one or the other cities having an academic, pharmacological or toxicological institution. Soon after its foundation, the SSPT became a national society member of the IUPHAR. Apart from IUPHAR, the SSPT collaborated with national societies including the German, French, British and Canadian organisations. The SSPT is also a member of the EPHAR and collaborates with the International Union of Toxicology and EUROTOX. In 1969, the SSPT became one of the founding members of the Union of Swiss Societies of Experimental Biology (now called 'Life Sciences Switzerland', LS2) and a member of SCNAT (Fig. 2).

In 2005, the SSPT was divided into three 'daughter' societies: The SSEP, the Swiss Society of Clinical Pharmacology and Toxicology (SSCPT) and the Swiss Society of Toxicology (SST). These societies created their own by-laws and councils, but remained members of the SSPT.
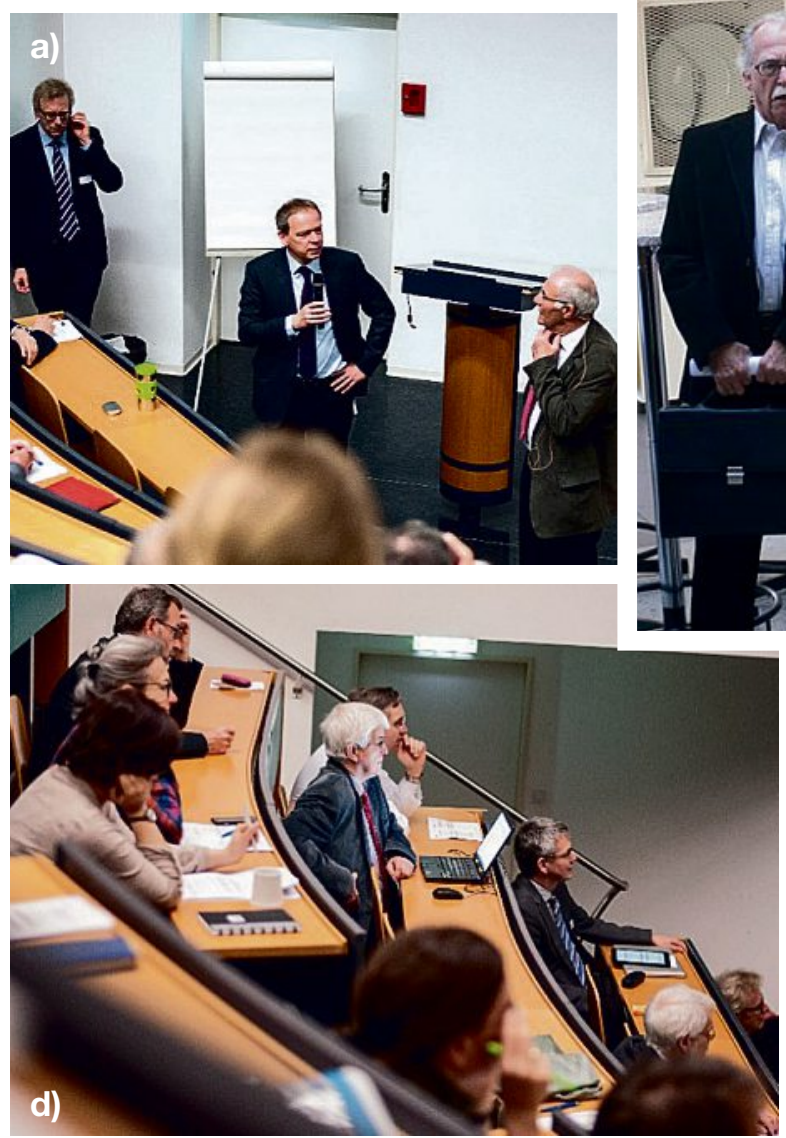

Fig. 4. Impressions of the $50^{\text {th }}$ Anniversary Meeting of the SSPT in 2015: a) (from left to right): Dr. Hugo Kupferschmidt, Tox Info Suisse, Zurich, Prof. Hans-Uwe Simon, University of Bern and Prof. em. Urs Rüegg, Universities Geneva and Lausanne; b) Prof. em. Urs Meyer, University of Basel (left) and Prof. em. Humphrey Rang, University of London, President of the British Pharmacological Society (right) c) Prof. em. Friedlieb Pfannkuch, Hoffmann-La Roche, Basel; d) auditorium view; ; e) Members of the SSPT council (from left to right) Dr. Martin Traber; Biogen, Zug;

Dr. Hugo Kupferschmidt, Tox Info Suisse, Zurich, PD Dr. Gabriele Weitz-Schmidt, AlloCyte Basel, Dr. Thomas Weiser, Hoffmann-La Roche, Basel and Prof. Dr. Stephan von Gunten, University of Bern.
This division led to an increase in memberships and gave the newly formed societies more independence and flexibility with regard to national and international first president of the SSEP. In 2006, the Swiss Society of Pharmaceutical Medicine joined the SSPT. To date, the SSPT repesents more than 500 members encompassing scientists from both academia and dustry.

2015, the SSPT - and thus the during the SSPT Spring Meeting held on April $23^{\text {rd }}$ in Bern (Fig. 4). The program presidential lectures given by professors Matthias Schwab (President of the German Society of Experimental and Pharmacology and Toxicology), Humphrey Rang (President of the British Pharmacological Society) and Dr. Hugo An overview on the history of the SSPT has been prepared by Urs Rüegg, professor

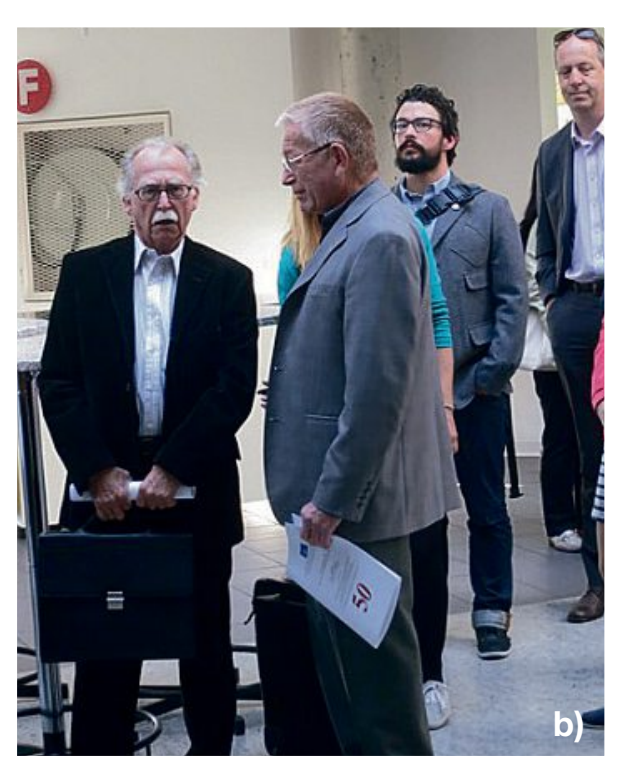
of the University Bern was elected as the
Geneva and Lausanne and published in 2015 in International Pharmacology. ${ }^{[3]}$

\section{Future Challenges of Experimental Pharmacology}

Despite the historical successes of the pharmaceutical industry in experimental pharmacology the future challenges of this discipline are severalfold: First and foremost, there is great concern about the decline of training and education in pharmacology, which seems to be in stark contrast to the fact that the demand for welltrained pharmacologists has hardly ever been higher. Pharmacology departments teaching experimental pharmacology with all its aspects are disappearing not only in Switzerland but also in UK, Germany and other countries. The consequence is that young scientists specialised in other scientific disciplines, e.g. molecular biology, are entering pharmacology without having been exposed, other than for their speciality, to the field before. These scientists bring interesting new aspects and valuable technologies into the field. However, their approaches to the drug discovery process have been described as more 're-

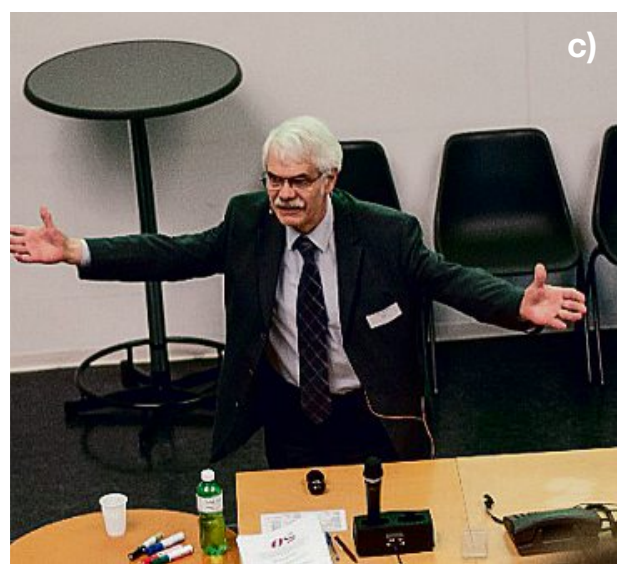

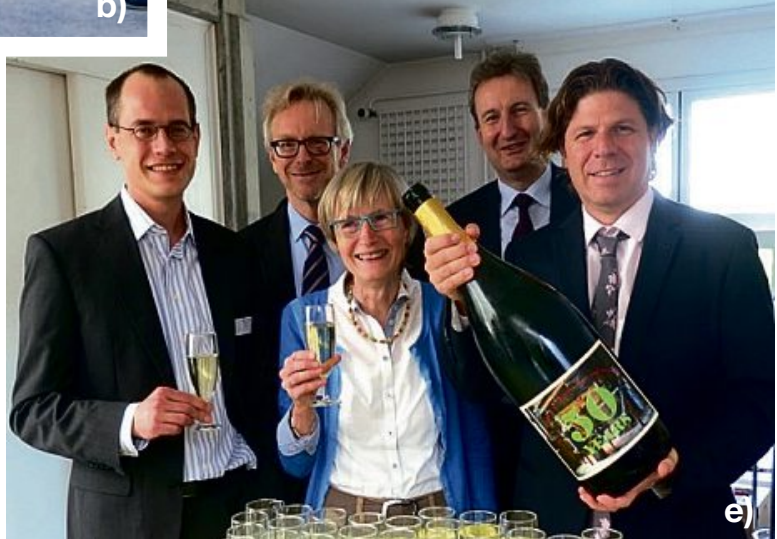


ductionist' rather than 'holistic', leaving the vital need for a 'holistic' approach and evolution of experimental pharmacology largely un-recognized and un-attended. ${ }^{[4]}$ Hand-in-hand with this undesirable development, a shortage of training in techniques involving the use of animals has been observed, ${ }^{[5]}$ although it is well established that drug development is critically dependent on the continued evolution of animal models predictive for human disease. ${ }^{[6]}$ Furthermore, the availability of animal models of high quality is widely accepted as a pre-requisite of reducing the total number of animal experiments in the future. In fact, the major cause of the high levels of failure of drug candidates during the clinical (rather than pre-clinical) development stage is their lack of efficacy. Interestingly, the lack of efficacy is most eminently noted for therapeutic areas in which animal disease models are largely non-predictive for human disease, such as oncology and CNS. ${ }^{6]}$ Consistent with these worrying trends, experimental pharmacology is also falling back with respect to modern learning technologies. So far, there is only sparse or no representation of topics relating to experimental pharmacology on websites offering free online courses such as www.edx.org or similar publicly accessible sites.[3]

Secondly, the unfortunate divide of academic experimental pharmacology and frequently firewalled industrial pharmacological research contributes to the current dilemma of experimental pharmacology. Clearly, closer interactions between academic and industrial institutions would be of great advantage to the field. Although numerous initiatives have been started in Switzerland to foster collaborations between industry and academia, the desired progress regarding industry-academia interactions has not been achieved yet. This is quite surprizing given the fact that most drugs are developed in the industrial environment, whereas at the same time novel therapeutic targets often emerge from academic research.

Last but not least experimental pharmacologists need to more assertively embrace the opportunities offered by newly emerging therapeutic modalities and concepts. The complexity inherent to the development of new forms of biopharmaceutical interventions represents new types of challenges not faced by traditional experimental pharmacology before. Accepting these challenges and embracing the associated opportunity will allow to newly define and widen the boundaries of experimental pharmacology in the future. Experimental pharmacology, still widely understood as the discipline which studies 'actions of drugs', may become newly launched as the discipline which takes charge of advanc- ing conventional 'drug therapy' to new horizons of integrated therapeutic interventions, continuously updating concepts for the needs of patients, caregivers and societies.

\section{The Way Forward}

In order to adequately advance the field of experimental pharmacology, the SSEP - together with the SSPT - has started to make universities in Switzerland aware of the challenges the discipline is facing, as well as of the unique opportunities experimental pharmacology could offer when supported adequately. Based on this initiative it is expected that educational programs addressing the needs of future experimental pharmacologists will be re-implemented and strengthened. Further, the SSEP - together with the SSPT - will continue to organize regular scientific meetings covering 'hot' topics in pharmacology, thus directly serving the educational needs of experimental pharmacologists. For example, this years' SSPT Spring Meeting focussed on pharmacological and toxicological aspects of novel modalities such as RNA-based therapeutics, gene and cell therapy. Participants' resonance was impressive, indicating that this type of meeting addresses an important unmet educational need and fills a major gap in current curricula. Addressing another need of similar importance for the future of experimental pharmacology, the next SSPT Spring Meeting, which will take place on April $20^{\text {th }}, 2017$ in the Inselspital in Bern is foreseen to review the current state-of-theart of animal experimentation.

With the aim to strengthen the professional profile of experimental pharmacologists, the SSEP/SSPT recently contacted EPHAR regarding the European Certified Pharmacologist (EuCP) program. This program has been initiated by EPHAR in 2014. Pharmacologists certified as EuCPs "will have proven that their competency profile, in addition to their personal specialised scientific expertise, covers expert knowledge in all major fields and that they have experiences and practical awareness in a wide spectrum of pharmacological techniques. The system guarantees that common high standards are applied for this certification by all participating societies of pharmacology throughout Europe". ${ }^{[7]}$ It is one future goal of the SSEP/SSPT to motivate and enable the participation of their members in this promising initiative and program.

In which ways can the SSEP promote industry-academia interactions? Traditionally, at least one member of the SSEP board comes from the pharmaceutical industry and represents the colleagues working in this environment. Further, scientists from academia and industry work together to determine the topics of scientific meetings organised or co-organised by the SSEP/SSPT and invite speakers both from academic and pharmaceutical industry. In addition, several seminar series at Swiss Universities have been initiated by members of the SSEP, covering numerous aspects of drug discovery and development. However, despite these initiatives, it is readily apparent that the perception of a divide between 'academic' and 'industrial' pharmacological research is deeply engrained in the minds and difficult to overcome. It will require a new sense of urgency to motivate the changes needed to successfully bridge current gaps between industry and academia in experimental pharmacology. This will allow to jointly advance the discipline to new horizons and to realize currently untapped synergies. The foundation of platforms promoting collaborations between academia and industry-based groups involved in drug discovery and development at the PharmaCenter of the University of Basel, or the formation of life science clusters between academic and industrial institutions in the area of translational medicine in Basel, Lausanne and Zurich may be first steps in the right direction. No time should be lost, however.

\section{Conclusions}

Traditional concepts of 'experimental pharmacology', artificially restricting the discipline to the discovery, research and development of conventional 'drugs', hinder the advancement of this key discipline to new horizons of innovative and more holistic therapeutic concepts. Internationally, experimental pharmacology in Switzerland appears to be exceptionally well-positioned to successfully overcome the hurdle and master the challenge. What is needed is continued strong advocacy by those concerned with the future of the discipline as well as its potential contribution to future economic and societal development. The SSEP provides a platform for professionals fascinated and attracted by this task.

Received: July 1, 2016

[1] Statistics about the number of medical prescriptions dispensed in the USA, 2016: http://www.statista.com/statistics/238702/us total-medical-prescriptions-issued/

[2] SSEP homepage: http://www.swisspharmtox. $c h / S S E P /$

[3] U. Rüegg, Int. Pharmacol. 2015, 84, 12.

[4] P. Vallance, T. G. Smart, Br. J. Pharmacol. 2006, 147, S304.

[5] M. J. A. Walker, M. L. M. Soh, Trends Pharmacol Sci. 2006, 27, 125.

[6] I. Kola, J. Landis, Nature Rev. Drug Discov. 2004, 3, 711

[7] EPHAR webpage: http://www.ephar.org/eucp/ index.html 